\title{
Spontaneous rupture of pyonephrosis presenting as anterior abdominal wall abscess: a rare case report
}

\author{
Kamlesh Hawaldar Singh ${ }^{*}$ (D) Ankit Vyas, Tarun Rochlani and Sujata Kiran Patwardhan
}

\begin{abstract}
Background: A pyonephrosis caused by an obstructing calculus is typically accompanied by fever, loin pain, and other signs of urinary tract infection. Occasionally, severe thinning of the renal parenchyma in pyonephrosis allows direct forniceal rupture into the retroperitoneum and very rarely into the anterior abdominal wall, misconstruing it as an isolated abdominal wall abscess.

Case presentation: Diabetes-related 55-year-old diabetic male presented with abscess in his periumbilical region extending into right lumbar region. He did not exhibit any urinary symptoms, and contrast enhanced computed tomography [CECT] abdominal and pelvic examinations revealed right pelvic calculus with pyonephrosis. There is a $7.5 \mathrm{~mm}$ defect in the lower pole of the right kidney with $171 \mathrm{cc}$ of collection adjacent to the kidney communicating with $150 \mathrm{cc}$ of superficial abdominal wall collection through a $15 \mathrm{~mm}$ defect. Incision and drainage of abdominal and retroperitoneal abscesses were done at first. Right DJ stenting was performed. Right lateral decubitus was placed for dependent drainage. Resolution of residual collections was confirmed by subsequent ultrasonography KUB, and drain was then removed. Right DJ stenting done, and patient was discharged. Two months later, DTPA scan done and revealed GFR of $30 \mathrm{ml} / \mathrm{min}$ of right kidney. Patient underwent right percutaneous nephrolithotomy.

Conclusion: The sudden rupture of pyonephrosis is a rare event. Even rarer is the presentation of pyonephrosis as an abscess on the anterior abdominal wall. The correct diagnosis and search for the source of the abscess must be undertaken before intervention. An aggressive and prompt management is needed to prevent further complications from occurring. This case is being presented to add to the literature with regard to abnormal presentations of ruptured pyonephrosis and its management.
\end{abstract}

Keywords: Pyonephrosis, Anterior abdominal wall abscess, Rupture, Spontaneous

\section{Background}

Moderate to severe hydronephrosis can uncommonly undergo spontaneous decompression following a calyceal rupture $[1,2]$. The rupture is usually a result of an obstructing calculus but may also result from posterior urethral valves, benign prostatic hyperplasia, pelvic tumors, and abdominal aortic aneurysm and retroperitoneal fibrosis $[3,4]$. There are times when the urine in

*Correspondence: dockamlesh4047@gmail.com

Department of Urology, SETH GSMC AND KEM Hospital, Parel, Mumbai 400012, India the collecting system is infected, producing pus (pyonephrosis) bursting into the perinephric space, psoas muscle, and quadratus lumborum muscle [5, 6]. A number of factors contribute to pyonephrosis, including immunosuppressive status and anatomic obstruction of the urinary tract, of which 75 percent is caused by kidney stones [7]. In pyonephrosis, antibiotics are ineffective unless the pus is surgically removed. Presented here is a rare case of spontaneous rupture of pyonephrotic kidney resulting from pelviureteric junction calculi presenting as anterior abdominal wall abscesses. 


\section{Case presentation}

A 55-year-old diabetic male presented to the emergency surgical unit with complaints of high grade fever with chills, right-sided abdominal pain, and swelling. The patient had been visiting local doctors for fever for one week prior to the current visit and receiving antibiotics without undergoing any laboratory or radiological testing. The patient did not have any urinary complaints in the past or present. Diabetic since 4 years, he was on Tab Metformin $500 \mathrm{mg}$ twice a day. $\mathrm{He}$ is a tobacco chewer since 20 years. On examination, he had a blood pressure of $110 / 60 \mathrm{mmHg}$, a pulse rate of 118 beats per minute, a respiratory rate of 29 breaths per minute and a body temperature of $39.0^{\circ} \mathrm{C}$. Upon examination, there was a $10 \mathrm{X} 12 \mathrm{~cm}$ soft, fluctuant \& tender swelling in the periumbilical region extending into the right lumbar region (Fig. 1a, b, c). There were raised leucocytes $(18,600$ cells/microliter of blood) and predominantly neutrophilia (over 90\%) along with anemia [ $\mathrm{Hb} 6.2]$. The levels of blood urea nitrogen and serum creatinine were within normal limits, at $11.3 \mathrm{mg} /$ $\mathrm{dl}$ and $1.2 \mathrm{mg} / \mathrm{dl}$, respectively. The random blood sugar reading was $180 \mathrm{mg} / \mathrm{dl}$. In urine microscopy, there were 3-4 red blood cells/high-power field and 100 pus cells/high-power field. On urine culture, cefosulbactam and amikacin were sensitive to coliform bacteria. A chest radiograph showed no significant abnormalities in the posteroanterior view. An abdomen and pelvis ultrasound revealed a $20 \mathrm{~mm}$ calculus in the lower calyx of the right kidney extending up into the renal pelvis, resulting in mild hydronephrosis with mobile internal echoes in the pelvicalyceal system along with perirenal collection, and subcutaneous collection. The CECT abdomen and pelvis revealed a calculus measuring $13 \times 25 \times 18 \mathrm{~mm}$ [HU 1400] in the lower pole with mild hydronephrosis. There is a defect of size $7.5 \mathrm{~mm}$ in the lower pole of the right kidney, which results in a collection of size $171 \mathrm{cc}$ that is communicating with the superficial collection in the subcutaneous plane of size $150 \mathrm{cc}$ through a defect of size $15 \mathrm{~mm}$ (Fig. 2a, b, c). There were no obvious abnormalities in the bony spine. There was no evidence of free fluid in the peritoneum. A ruptured pyonephrosis was diagnosed. The abdominal and retroperitoneal abscesses were incised and drained. The patient was kept in right lateral decubitus for dependent drainage. On subsequent ultrasonography KUB, residual collections were confirmed to be gone, and the drain was removed. Patient was discharged after right DJ stenting. A DTPA scan was performed two months later that revealed a GFR of $30 \mathrm{ml} /$ min for the right kidney. Patient underwent right percutaneous nephrolithotomy. The six-month follow-up was uneventful. (a)

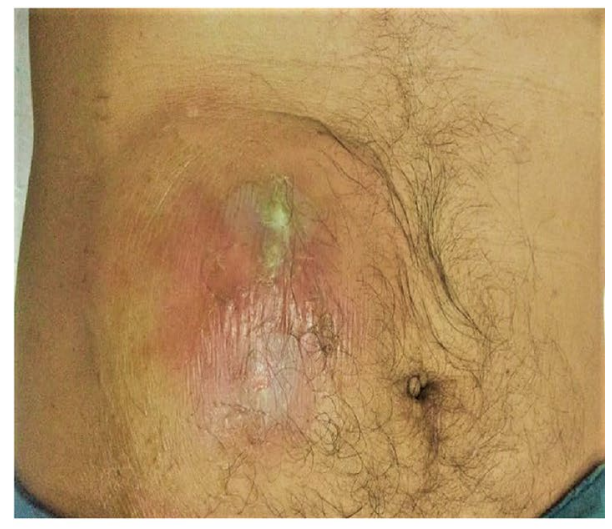

(b)

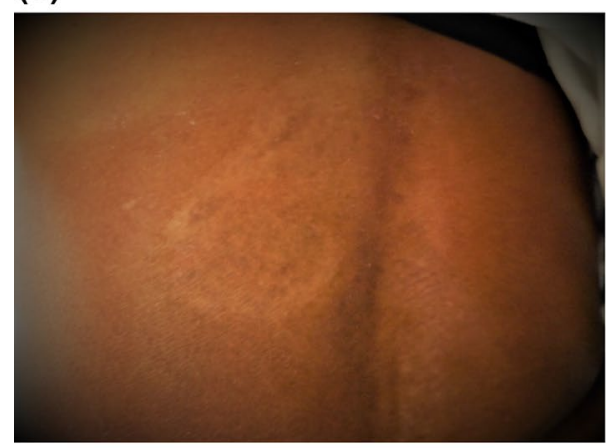

(c)

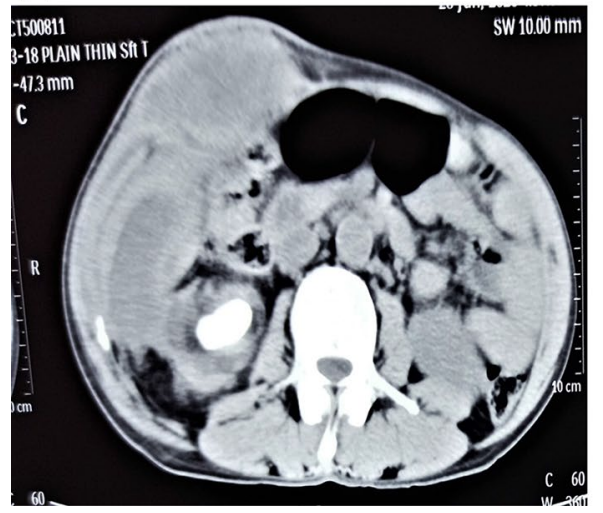

Fig. 1 a Supine position. b Prone position. c CT depicting the abscess

\section{Discussion}

More commonly, kidney ruptures occur at the site of the renal parenchyma over the renal pelvis [8]. Rarely, forniceal rupture occurs in patients with pyelonephritis or pyonephrosis, especially if there is an obstruction [9]. Due to lumbodorsal fascia, ruptured pyonephrosis usually manifests as retroperitoneal abscess. However, in our case, it manifested as an anterior abdominal wall 


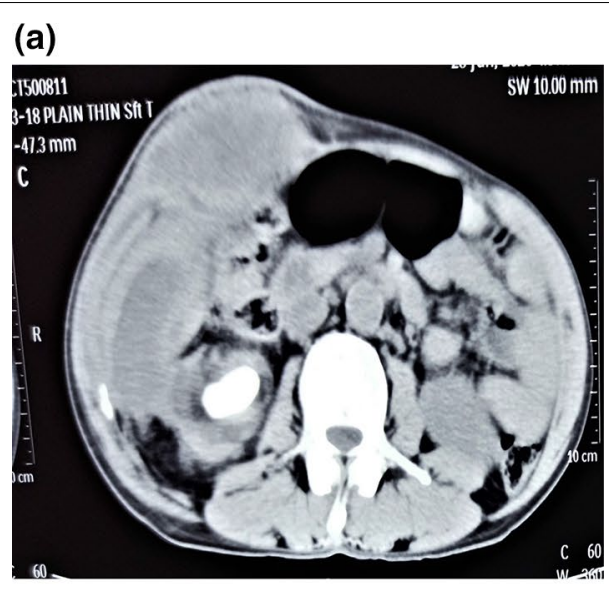

(b)

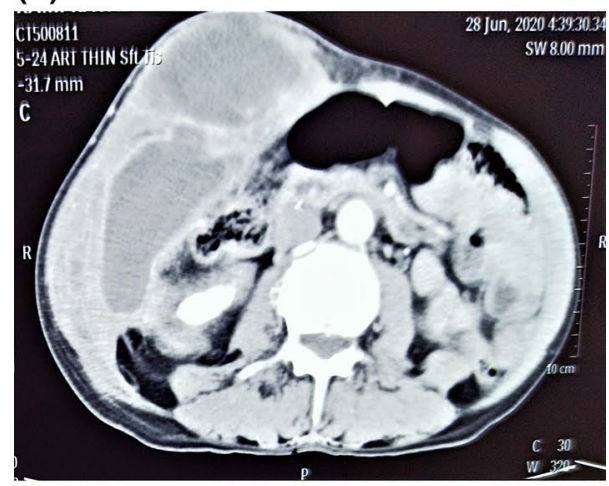

(c)

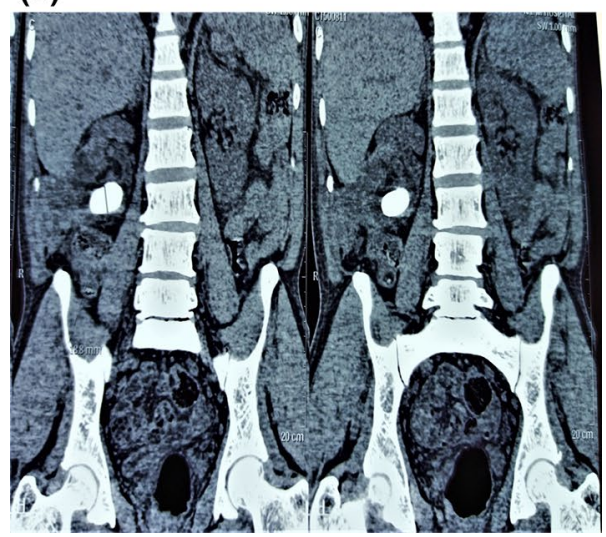

Fig. 2 a Transverse axial plain CT cut section showing communicating perirenal and subcutaneous abscess along with lower calyceal renal calculus. b Transverse axial arterial phase CT cut showing communicating perirenal and subcutaneous abscess. c Coronal CT cut section showing right lower calyceal stone with defect in renal parenchyma

abscess. An explanation for the defect depicted in the CT scan was not available. However, a nephrocutaneous fistula corresponding to the spontaneous development of an abnormal communication between the kidney and the skin, crossing through the retroperitoneum and abdominal wall structures following the lowest resistance points such as the Petit's triangle and the Grynfeld quadrilateral can be considered. Also differentials could be xanthogranulomatous pyelonephritis or emphysematous pyelonephritis, but CT scan picture suggests less likely of them being differentials. It is imperative to take prompt action by draining urine and diverting it. According to the current literature, percutaneous nephrostomy [5] or ureteral stents, along with pus drainage, should be performed to prevent septic shock in patients. An incision and drainage were successfully performed following CT scan demonstration of both abscesses communicating. Additionally, no difficulty was encountered during percutaneous nephrolithotomy. Our case suggests that a delayed definitive management is a good idea. Based on an extensive literature search, only six cases have been reported of spontaneous rupture of the pyonephrosis combined with formation of a psoas abscess $[5,10]$. We found an anterior abdominal wall abscess in our case, a finding not reported in the literature. A lumbar panniculitis associated with subcutaneous abscess associated with a pyonephrosis case has been reported, however [11]. The literature also describes cases of peritoneal rupture of pyonephrosis causing peritonitis and splenic abscess [12, 13].

\section{Conclusion}

Spontaneous rupture of Pyonephrosis is a rare occurrence. Rarer is the presentation of Pyonephrosis as anterior abdominal wall abscess. Accurate diagnosis and thorough search for source of abscess must be considered prior to intervention. CT abdomen should form part of usual protocol for evaluation of all lumbar abscesses to rule out renal cause of abdominal abscess. Prompt and aggressive management is needed to prevent further complication. This rare case is presented in order to add to the literature, the abnormal presentations of ruptured pyonephrosis and its management.

\section{Abbreviations \\ CECT: Contrast enhanced commuted tomography; DJ: Double J stent; KUB: Kidney, ureter, bladder; GFR: Glomerular filtration rate; DTPA: Diethylenetri-} aminepentaacetic acid; HU: Hounsfield unit.

\section{Acknowledgements}

We thank our patient for allowing us to share his details, the radiologist for providing valuable help in diagnosing the condition and the co-authors for their expertise and assistance throughout the process. I intend to submit the manuscript to 'AFRICAN JOURNAL OF UROLOGY', and it is not currently under consideration elsewhere.

\section{Authors' contributions}

KS-Corresponding author- Idea of case report, literature search, data collection, case report writing AV, TR-Figure editing , literature search 
SKP_Checked the manuscript and made corrections All authors read and approved the final manuscript.

\section{Funding}

No funding required.

\section{Availability of data and materials}

Data sharing is not applicable to this article as no datasets were generated or analyzed during the case report making.

\section{Declarations}

Ethics approval and consent to participate

Our institution does not require ethical approval for this case report.

\section{Consent for publication}

Written informed consent was obtained from the patient for publication of this case report and accompanying images.

\section{Competing interests}

The authors declare that they have no competing interests.

Received: 10 May 2021 Accepted: 9 August 2021

Published online: 17 August 2021

\section{References}

1. Koktener A, Unal D, Dilmen G, Koc A (2007) Spontaneous rupture of the renal pelvis caused by calculus: A case report. J Emerg Med 33: 127-129. https://bit.ly/2SF0X39

2. Patel N, Thanvi R, Jain A, Sathvara P (2015) Devastating complication due to rupture of obstructive perinephric urinoma with secondary pyonephrosis necessitating nephrectomy of nonfunctional kidney in a child. Archives of Medicine \& Health Sciences 3: 314-316. https://bit.ly/2Yyzt2X

3. Singh I, Joshi M, Mehrotra G (2009) Spontaneous renal forniceal rupture due to advanced cervical carcinoma with obstructive uropathy. Arch Gynecol Obstet 279: 915-918. https://bit.ly/3d8GL11
4. Lien WC, Chen WJ, Wang HP, Liu KL, Hsu CC (2006) Spontaneous urinary extravasation: An overlooked cause of acute abdomen in ED. Am J Emerg Med 24: 347-349. https://bit.ly/3aUAZPk

5. Patil V, Ichalakaranji R, Patil SB, Pattar R, Ashrith IM (2014) Spontaneous rupture of a calculus pyonephrotic kidney into the retroperitoneal cavity presenting as psoas abscess-A Case Report. Int J Biomed Adv Res

6. : 262-263. 6. Erol A, Coban S, Tekin A (2014) A giant case of pyonephrosis resulting from nephrolithiasis. Case Rep Urol 2014: 161640. https://bit.ly/ $35 \mathrm{jGs} 0 \mathrm{Z}$

7. Chen YL, Wang MC, Huang JZ (2008) Pyonephrosis Kidney Dial 20:14-18

8. Quaresima et al (2011) Spontaneous intraperitoneal rupture of pyonephrosis in a patient with unknown kidney carcinosarcoma: a case report. World J Surg Oncol 9:39

9. Jalbani IK, Ather M (2014) Renal forniceal rupture in pregnancy secondary to obstructive renal stone presenting with acute renal failure. Saudi J Kidney Diseas Transpl 25(5):1081

10. Rastogi R, Neha PV, Meena GL (2020) Pyonephrosis presenting as lumbar abscess-an uncommon clinicoradiological entity. Arch Clin Nephrol 6(1): 017-019. https://doi.org/10.17352/acn.000041

11. Pauwels C, Bulai-Livideanu C, Chiavassa H, Lamant L, et al. (2009) Lumbar panniculitis with subcutaneous abscess revealing pyonephrosis. Ann Dermatol Venerol 136: 727-729. https://bit.ly/2VTjYka

12. Hendaoui MS, Abed A, M'Saad W, Chelli H, Hendaoui L (1996) A rare complication of renal lithiasis: peritonitis and splenic abscess caused by rupture of pyonephrosis]. J Urol (Paris) 102: 130-133. https://bit.ly/2VWlu IP

13. Jalbani IK, Khurrum M, Aziz W (2019) Spontaneous rupture of pyonephrosis leading to pyoperitoneum. Urol Case Rep 31; 26:100928. https://bit.ly/ $2 \times n F Q L$

\section{Publisher's Note}

Springer Nature remains neutral with regard to jurisdictional claims in published maps and institutional affiliations.

\section{Submit your manuscript to a SpringerOpen ${ }^{\circ}$ journal and benefit from:}

- Convenient online submission

- Rigorous peer review

- Open access: articles freely available online

- High visibility within the field

- Retaining the copyright to your article

Submit your next manuscript at $\boldsymbol{\nabla}$ springeropen.com 